\title{
Infecções relacionadas à assistência à saúde ocorridas em uma Unidade de Terapia Intensiva
}

\author{
Infections to assistance to health occurred at an Intensive Care Unit \\ Cianna Nunes Rodrigues ${ }^{1}$, Dagolberto Calazans Araújo Pereira²
}

\begin{abstract}
Resumo: As infecções hospitalares são as mais importantes complicações ocorridas em pacientes hospitalizados. Objetivou-se descrever as infecções relacionadas à assistência à saúde ocorridas em uma UTI de um hospital público em São Luís-MA. Trata-se de um estudo descritivo, analítico e transversal realizado com 1048 pacientes que foram admitidos na UTI no período de janeiro de 2012 a dezembro de 2013. Para análise estatística foi utilizado o Programa Bioestat 5.0, sendo usado o Teste do Qui-quadrado estimando a Odds Ratio (OR), intervalo de confiança de $95 \%$ e valor $p<0,001$, e o Teste Kruskal-Wallis. Dos pacientes analisados, $50,38 \%$ eram do sexo feminino; $37,11 \%$ tinham mais de 65 anos; $43 \%$ foram internados para realizar cirurgia eletiva; $48,5 \%$ foram oriundos do centro cirúrgico; a duração média das internações foi de 7,41 dias e 31,25\% evoluíram ao óbito. Em relação aos eventos infecciosos ocorridos, 47,03\% apresentaram PAVM, 15,14\% pneumonia nosocomial, $14,05 \%$ ITU associada ao cateter e 9,73\% tiveram IPCS. Em se tratando dos óbitos ocorridos, os pacientes com PAVM têm uma chance 4 vezes maior de evoluírem a óbito do que os pacientes que não apresentaram nenhum tipo de infecção $(p<0,0001)$; e, para finalizar, os pacientes com PAVM apresentam uma maior probabilidade de desenvolver os quadros de sepse e choque séptico do que os pacientes que apresentaram ITU $(p=0,008)$. Constatou-se que a infecção mais incidente foi a PAVM, o que sugere uma reavaliação nos protocolos e educação permanente dos profissionais de saúde.
\end{abstract}

Palavras-chaves: Infecções. Assistência. Unidade Terapia Intensiva.

\begin{abstract}
The hospital infections are the most important complications occurred among hospitalized patients. It is aimed at describing infections related to the health assistance occurred at an UTI of a public hospital in São Luís-MA (Brazil). It dealt with a descriptive, analytical and transversal study performed among 1048 patients who were admitted in the UTI in the period from January 2012 to December 2013. To the statistical analysis it was used the Bioestat Software 5.0, being used the QuiSquare Test. It is believed that the Odds Ratio (OR) in an interval of confidence $95 \%$ and as to its value $\mathrm{P}<0,0001$, and the Kruskal-Wallis Test. From the analysed patients $50,38 \%$ were female ones; $37,11 \%$ were more than 65 years old; $43 \%$ were admitted to have elective surgery; $48,5 \%$ came from the chirurgical centre; the leught of hospitalization was of 7,41 days and $31,25 \%$ died. As to the infections occurrences $47,03 \%$ presented PAVM, $15,14 \%$ got nosocomial pneumonia, $14,05 \%$ ITU associated to catheter and $9,73 \%$ got IPCS. As to the occurred deaths the PAVM pacients had a 4 time chance major to face death than the patients who didn't expressed any type of infection $(p<0,0001)$; and to finish the PAVM patients expressed a major probability of developing the septic process and septic shock than the patients who expressed ITU $(p=0,008)$. It was observed that the most frequent infection was the PAVM, that suggests a reassessment of the protocols and a continuing education for the health professionals.
\end{abstract}

Keywords: Infection. Assistance. Intensive Cure Unity.

1 Mestranda do Mestrado em Gestão de Programas e Serviços de Saúde- Universidade Ceuma 2 Docente do Mestrado em Gestão de Programas e Serviços de Saúde - Universidade Ceuma 


\section{Introdução}

Nos dias atuais, as Infecções relacionadas à Assistência à Saúde (IRAS) atingem com frequência os sistemas de saúde, constituindo-se como um grave problemas de saúde pública mundial, elevando os custos hospitalares e os índices de morbidade e mortalidade entre os pacientes. ${ }^{1}$

As IRAS são definidas como toda e qualquer infecção que acomete o indivíduo, seja em instituições hospitalares, seja em atendimentos ambulatoriais na modalidade de hospital dia ou domiciliar, e que possa estar associada a algum procedimento assistencial, seja ele terapêutico ou diagnóstico? ${ }^{2}$.

Estima-se que nos Estados Unidos, anualmente, ocorram cerca de 1.7 milhões de casos de infecções relacionadas à assistência à saúde, com registros de cerca de 100.000 óbitos relacionados às IRAS ${ }^{3}$. No Brasil, apesar de não haver de dados, estima-se que aproximadamente 5 a $15 \%$ dos pacientes hospitalizados e 25 a $35 \%$ dos pacientes admitidos em UTI adquiram algum tipo de infecção relacionada à assistência à saúde sendo ela, a quarta causa de mortalidade. ${ }^{4-5}$

A Organização Mundial de Saúde (OMS), considera que 1,4 milhão de infecções ocorrem a qualquer momento, tanto em países desenvolvidos quanto em desenvolvimento. Nos Estados Unidos, estima-se que cerca de 2 milhões de IRAS ocorram anualmente, o que resulta em 60 a 90 mil mortes com um custo aproximado de 17 a 29 bilhões de dólares. Em média, de 5 a $15 \%$ de todos os pacientes internados desenvolvem IRAS e no Brasil, em virtude da ausência de sistematização de informações não se dispõe de estimativas precisas dessas infecções. ${ }^{6}$

Com a introdução de novos métodos diagnósticos e terapêuticos e o avanço tecnológico na assistência à saúde, aumentam-se as possibilidades de intervenção e diminuição da mortalidade. Entretanto, tal avanço traz um aumento do risco de IRAS e deste modo controlar a ocorrência dessas infecções é uma tarefa desafiadora. $^{7}$

Nas Unidades de Terapia Intensiva (UTI), as IRAS estão associadas principalmente ao uso de procedimentos invasivos (cateteres venosos centrais, sondas vesicais de demora, ventilação mecânica e outros), imunossupressores; prolongado período de internação, colonização por microrganismos resistentes, uso indiscriminado e abusivo de antimicrobianos e o próprio ambiente da unidade que já favorece a seleção natural de microrganismos e, consequentemente a colonização e/ou infecção pelos mesmos, inclusive os microrganismos multirresistentes. ${ }^{3-8-9}$

Um fator de risco para infecção hospitalar é simplesmente um indicador de risco, ou um fator associado à infecção hospitalar. Tal indicador de risco não precisa necessariamente ser a causa da infecção ou preceder a infecção. De acordo com a Lei o 7498 de 25 de junho de 1986, que dispôs sobre a regulamentação do exercício da Enfermagem, cabe ao enfermeiro, enquanto integrante da equipe de saúde, a prevenção e o controle sistemático da infecção nosocomial e de doenças transmissíveis em geral. ${ }^{10}$

Em face do contexto, torna-se pertinente a realização de estudos que apresentem as taxas de infecções ocorridos nas UTI, visando relacionar 
a ocorrência das infecções com a assistência à saúde.

Os resultados dessas IRAS possibilitam 0 conhecimento da realidade da UTI com relação a ocorrência de infecções, aos fatores relacionados à assistência à saúde e às medidas necessárias para redução e prevenção da dessas infecções.

O objetivo desta pesquisa foi descrever a ocorrência de infecção relacionada à assistência à saúde na UTI de um hospital público de alta complexidade e referência para ensino em São Luís-MA.

\section{Material e Método}

Tratou-se de um estudo descritivo, analítico e transversal realizado em uma UTI de um hospital da rede pública, de médio porte e alta complexidade situado no município de São Luís-MA, que possui convênio com instituições de ensino superior do setor público e privado.

Diz respeito a de uma UTI com 11 leitos, sendo um deles destinados para isolamento e que atende a todas as especialidades médicas e cirúrgicas.

Foram analisados os dados de 1048 pacientes admitidos na UTI referida, no período de janeiro de 2012 a dezembro de 2013, e que estavam disponíveis em um banco de dados.

Inicialmente, foi realizada uma análise exploratória e descritiva para se obter as características da população estudada em relação as variáveis demográficas (gênero e faixa etária) e clínicas (tipo de internação, origem dos pacientes, duração da internação e o desfecho desses pacientes na unidade). Em seguida, foram descritos os eventos infecciosos ocorridos na UTI no período pesquisado, as infecções relacionadas à assistência à saúde, os óbitos ocorridos em decorrência dessas infecções e a relação entre essas infecções e o desenvolvimento de sepse, sepse grave e choque séptico.

As informações para este estudo foram conseguidas através dos registros existentes em um banco de dados alimentado pela coordenação médica da unidade. Este banco de dados é gerado através de um software de gestão hospitalar chamado Epimed que permite gerenciar informações clínicas e epidemiológicas, e produzir relatórios em tempo real que, através destes, auxiliarão na análise de indicadores e melhora da qualidade do atendimento hospitalar. A coleta dos dados ocorreu no período de abril a junho de 2014, nos dias úteis da semana, no turno matutino, para realizar a coleta.

A análise estatística foi realizada através do programa estatístico Bioestat 5.0, sendo usado o Teste do Qui-quadrado estimando a Odds Ratio (OR), intervalo de confiança de 95\% e valor $P<0,001$ para as variáveis IRAS relacionadas à mortalidade, e o Teste Kruskal-Wallis foi utilizado para as variáveis IRAS relacionadas à ocorrência de sepse, sepse grave e choque séptico.

Conforme a Resolução 466/2012 do Conselho Nacional de Saúde (CNS), esta pesquisa foi aprovada pelo Comitê de Ética e Pesquisa (CEP) da Universidade CEUMA, por meio do parecer no 539739, de 24 de fevereiro de 2014.

\section{Resultados}

Foram analisados 1048
pacientes internados na UTI
referenciada, no período de janeiro de
2012 a dezembro de 2013 , e destes,
$528(50,4 \%)$ eram do sexo feminino e
$389(37,11 \%)$ estavam acima de 65


anos. No que se refere ao tipo de internação, observou-se que 451 (43\%) dos pacientes foram internados para realizar cirurgias eletivas; em relação a procedência 508 (48,5\%) foram oriundos do centro cirúrgico; a duração média de internação de foi de 7,41 dias e quanto ao desfecho deses pacientes na unidade $67,71 \%$ receberam alta e $31,25 \%$ evoluíram ao óbito.

Tabela 1: Distribuição dos pacientes internados na UTI no período de janeiro de 2012 a dezembro de 2013, quanto as variáveis demográficas e clínicas. São Luís/MA, 2015.

\begin{tabular}{|c|c|c|}
\hline Variáveis & $\mathbf{n}$ & $\%$ \\
\hline \multicolumn{3}{|l|}{ Sexo } \\
\hline Masculino & 520 & 49,6 \\
\hline Feminino & 528 & 50,4 \\
\hline \multicolumn{3}{|l|}{ Faixa etária } \\
\hline$<18$ & 32 & 3,1 \\
\hline $18-44$ & 280 & 26,7 \\
\hline $45-64$ & 347 & 33,1 \\
\hline$>65$ & 389 & 37,1 \\
\hline \multicolumn{3}{|l|}{ Tipo de internação } \\
\hline Clínica & 442 & 42,2 \\
\hline Cirurgia eletiva & 451 & 43,0 \\
\hline Cirurgia de urgência/emergência & 144 & 13,7 \\
\hline Não Informado (NI) & 11 & 1,0 \\
\hline \multicolumn{3}{|l|}{ Origem dos pacientes } \\
\hline Enfermaria/Quarto & 190 & 18,1 \\
\hline Emergência & 43 & 4,1 \\
\hline Centro Cirúrgico & 508 & 48,5 \\
\hline Transferência externa & 224 & 21,4 \\
\hline Outras unidades & 83 & 7,9 \\
\hline Duração média da internação (dias) & 7,41 & \\
\hline \multicolumn{3}{|l|}{ Desfecho na Unidade } \\
\hline Alta & 715 & 68,2 \\
\hline Óbito & 330 & 31,5 \\
\hline $\mathrm{NI}$ & 11 & 1,0 \\
\hline Total & 1048 & 100,0 \\
\hline
\end{tabular}


Analisando a tabela 2, observouse que dos 1048 pacientes internados no período pesquisado, $185(17,65 \%)$ apresentaram eventos de infecciosos dos quais, $87(47,03 \%)$ apresentaram PAVM, $28 \quad(15,14 \%)$ pneumonia nosocomial, $26 \quad(14,05 \%) \quad$ ITU associada ao cateter vesical, 18 $(9,73 \%)$ desenvolveram IPCS associadas ao cateter, 16 (8,65\%) apresentaram infecção relacionada ao acesso vascular (exceto IPCS) e 10 $(5,40 \%)$ desenvolveram outras infecções.

Com base nos dados demonstrados na tabela 3 , observouse que, em relação aos 510 pacientes que foram expostos à ventilação mecânica (48,30\%), a duração da VM em dias por paciente apresentou uma média de 8,92 , um desvio padrão de 8,61 e a densidade de incidência de PAVM foi de 15,83 . Em relação ao uso do cateter vesical, 916 utilizaram cateter vesical de demora $(86,74 \%)$, a permanência dos cateteres vesicais (dias) por paciente apresentou uma média de 6,60 , um desvio padrão de
$7,59 \%$ e a densidade de incidência de Infecção do trato urinário (ITU) no período foi de 3,48 . E, para finalizar, 764 (72,35\%) dos pacientes internados foram expostos com cateter vascular central (CVC), a permanência dos CVC (dias) por paciente apresentou uma média de 8,10, um desvio padrão de $8,84 \%$ e a densidade de incidência de Infecção da corrente sanguínea (ICS) primária com comprovação laboratorial no período foi de 2,91.

De acordo com a tabela 4, no que diz respeito ao desfecho dos pacientes com IRAS, pôde-se observar que os que apresentaram IPCS tiveram uma chance de quase 7 vezes maior de ir a óbito do que os pacientes que não apresentaram IRAS $(p=0,0001)$ os que tiveram ITU apresentaram uma chance 3 vezes maior $(p=0,007)$ e os que desenvolveram PAVM uma chance de 4 vezes maior $(p<0,0001)$ de irem a óbito do que os que não apresentaram IRAS.

Tabela 2: Distribuição das principais infecções hospitalares ocorridas na UTI no período de janeiro de 2012 a dezembro de 2013. São Luís/MA, 2015.

\begin{tabular}{lcc}
\hline \multicolumn{1}{c}{ Variáveis } & $\mathbf{n}$ & $\%$ \\
\hline Total de eventos infecciosos ocorridos no período & 185 & 100 \\
Foco da infecção & & \\
Pneumonia associada à ventilação mecânica (PAVM) & 87 & 47,0 \\
Pneumonia & 28 & 15,1 \\
Infecção do trato urinário (ITU) associada ao cateter & 26 & 14,1 \\
Infecção da corrente sanguínea (ICS) primária associada & & \\
ao cateter & 18 & 9,7 \\
Relacionada ao acesso vascular (exceto ICS) & 16 & 8,6 \\
Outras infecções & 10 & 5,4 \\
\hline
\end{tabular}


Tabela 3: Distribuição das infecções relacionadas à assistência à saúde ocorridas na UTI no período de janeiro de 2012 a dezembro de 2013. São Luís-MA, 2015.

\begin{tabular}{|c|c|c|}
\hline Variáveis & $\mathbf{n}$ & $\%$ \\
\hline \multicolumn{3}{|l|}{ Utilização da Ventilação Mecânica (VM) } \\
\hline Pacientes em VM no período $(\mathrm{n}, \%)$ & 510 & 48,3 \\
\hline Duração da VM (dias) por paciente (média \pm DP) & $8,92 \pm 8,61$ & \\
\hline $\begin{array}{l}\text { Densidade de incidência de PAVM (no de } \\
\text { eventos/1000dias de VM) }\end{array}$ & 15,83 & \\
\hline \multicolumn{3}{|l|}{ Utilização de cateter vesical (CV) } \\
\hline Pacientes com cateter vesical (CV) no período $(n, \%)$ & 916 & 86,74 \\
\hline Permanência dos CV (dias) por paciente (média \pm DP) & $6,6 \pm 7,6$ & \\
\hline $\begin{array}{l}\text { Densidade de incidência de ITU no período ( } n^{\circ} \text { de } \\
\text { eventos } / 1000 \text { cateteres dia) }\end{array}$ & 3,48 & \\
\hline \multicolumn{3}{|l|}{ Utilização de cateter vascular central (CVC) } \\
\hline Pacientes com cateter ou acesso vascular central $(n, \%)$ & 764 & 72,35 \\
\hline Permanência dos CVC (dias) por paciente (média $\pm \mathrm{DP}$ ) & $8,1 \pm 8,8$ & \\
\hline $\begin{array}{l}\text { Densidade de incidência de ICS primária comprovada } \\
\text { laboratorialmente no período (no de eventos } / 1000 \\
\text { cateteres dia) }\end{array}$ & 2,91 & \\
\hline
\end{tabular}

Tabela 4: Distribuição das IRAS ocorridas na UTI no período de janeiro de 2012 a dezembro de 2013, relacionadas ao desfecho desses pacientes na unidade. São Luís/MA, 2015.

\begin{tabular}{lccccccccc} 
& \multicolumn{2}{c}{ Alta } & \multicolumn{2}{c}{ Óbito } & \multicolumn{2}{c}{ Não } \\
& $\mathbf{N}$ & $\%$ & $\mathbf{N}$ & $\%$ & $\mathbf{N}$ & $\%$ & & & \\
& $\mathbf{N}$ & $\%$ & $\mathbf{N}$ & $\%$ & & & OR \\
\hline Sem IRAS & 667 & 66,5 & 255 & 25,4 & 6 & 0,6 & - & - & - \\
IPCS & 5 & 23,8 & 13 & 61,9 & 3 & 14,3 & 6,78 & 2,4 a 21,38 & 0,0001 \\
ITU & 12 & 46,2 & 14 & 53,8 & 1 & 3,8 & 3,05 & 1,37 a 6,83 & 0,007 \\
PAVM & 31 & 38,7 & 48 & 60,0 & 1 & 1,3 & 4,05 & 2,5 a 6,5 & $<0,0001$ \\
\hline
\end{tabular}

Os resultados demonstrados na tabela 5 revelam que os pacientes que apresentaram PAVM tem uma maior probabilidade de desenvolver os quadros mais graves de sepse (sepse grave e choque séptico) do que os pacientes que apresentaram ITU $(p=$
0,008). Não houve diferença de gravidade para ocorrência de sepse, sepse grave e choque séptico entre os pacientes com ICS quando comparados com ITU $(p=0,12)$, e com PAVM $(p=0,57)$. 
Tabela 5: Distribuição das IRAS ocorridas na UTI no período de janeiro de 2012 a dezembro de 2013, relacionadas à ocorrência de sepse, sepse grave e choque séptico. São Luís-MA, 2015.

\begin{tabular}{lcccccc}
\hline & \multicolumn{2}{c}{ IPCS } & \multicolumn{2}{c}{ ITU* $^{*}$} & \multicolumn{2}{c}{ PAVM $^{*}$} \\
\cline { 2 - 7 } & $\mathbf{n}$ & $\%$ & $\mathbf{n}$ & $\%$ & $\mathbf{n}$ & $\%$ \\
\hline Sepse & 9 & 42,8 & 18 & 69,2 & 32 & 40 \\
Sepse Grave & 10 & 47,6 & 7 & 26,9 & 35 & 43,7 \\
Choque Séptico & 1 & 4,7 & 0 & 0 & 11 & 13,7 \\
\hline
\end{tabular}

* - Diferença estatística $(p=0,008)$. Teste utilizado Kruskal-Wallis

\section{Discussão}

Em relação às variáveis gênero, faixa etária, tipo de internação e procedência dos pacientes, não foi realizada a associação dos mesmos com a ocorrência das infecções, tendo em vista que o banco de dados utilizado não forneceu essas informações.

$\mathrm{Em}$ um estudo realizado numa UTI de um Hospital Universitário da Bahia, mostrou-se que, de 1.142 pacientes analisados, $50,61 \%$ eram do sexo feminino, o que corrobora com os resultados encontrados neste estudo; em relação à faixa etária a maioria encontrava-se com idade maior ou igual a 50 anos, $80 \%$ da população era oriunda de sua residência e $27,06 \%$ foram a óbito, o que diverge dos resultados encontrados nesta pesquisa. ${ }^{11}$

Conforme o estudo realizado em uma UTI de um hospital universitário brasileiro, a média global de permanência nesse setor foi de 5,7 dias, a média para pacientes que não desenvolveram $\mathrm{IH}$ foi de 3,7 dias e de 19,3 dias para aqueles que desenvolveram infecção. A maioria dos pacientes utilizam procedimentos invasivos, dentre os quais sonda vesical de demora, cateter venoso central e ventilação mecânica. ${ }^{12}$

Sabe-se que a mortalidade nas Unidades de Terapia geralmente é elevada, sendo registradas taxas que variam de $9 \%$ a $38 \%$, dependendo da UTI, do perfil dos pacientes atendidos, principalmente relacionado à severidade clínica. E, quando se refere ao óbito ocorrido em pacientes que desenvolveram IRAS, essa taxa poderá chegar a $70 \% .{ }^{13}$

Em uma pesquisa realizada na UTI do Complexo Hospitalar Mangabeira Governador Tarcísio Burity, localizado no município de João Pessoa-PB, dos pacientes analisados $51,6 \%$ eram do sexo masculino; em relação ao tipo de internação $57,4 \%$ foram indicações clínicas; quanto a procedência $38,9 \%$ foram procedentes de outras unidades do hospital; o tempo médio de permanência na UTI foi de 9,18 dias, o que diverge com os dados encontrados no presente estudo; e no que se refere à faixa etária, $52 \%$ estavam acima de 65 anos. Este dado corrobora com esta pesquisa. ${ }^{14}$

Os fatores que predispõem os indivíduos à infecção ligam-se ao estado de saúde de cada um, bem como aos métodos invasivos atinentes aos aparelhos e procedimentos de tratamento, envolvendo transplantes, 
transfusões,

fatores

imunodepressivos, uso de ventilação invasiva, nutrição parenteral, abuso da utilização de antibióticos, betabloqueadores e histamínicos, uso de cateteres e outros equipamentos. ${ }^{15}$

De acordo com um estudo realizado em uma UTI de um hospital universitário brasileiro, num total de 1.886 pacientes admitidos na UTI, a taxa de infecção hospitalar foi de $20,3 \%$ em um total de 246 pacientes. $^{12}$

Em um estudo realizado num hospital universitário da Bahia, ao serem estudados os sítios de $\mathrm{IH}$, foi observado que $43,52 \%$ adquiriram pneumonia hospitalar e os demais $56,48 \%$ apresentaram outros tipos de $\mathrm{IH}$, associados ou não a mais de um sítio de $\mathrm{IH}$ assim distribuídos: $26,09 \%$ infecção do sítio cirúrgico, 22,59\% ITU, $12,26 \%$ desenvolveram sepse, $5,52 \%$ infecções cutâneas, 2,80 infecções indeterminadas e $3,5 \%$ outros sítios de $\mathrm{IH}^{11}$

Em Minas Gerais foi realizado um estudo relacionado aos procedimentos invasivos realizados na UTI adulto do Hospital Aroldo Tourinho, situado na cidade de Montes Claros, com o perfil das infecções hospitalares. $O$ estudo mostrou que 0 procedimento predominantemente associado à infecção foi 0 tubo orotraqueal que é utilizado na ventilação mecânica invasiva com $92,85 \%$ de incidência, seguido de $7,14 \%$ relacionados ao cateter venoso central, evidenciando o pulmão como a topografia mais acometida pelas infecções hospitalares, especialmente a pneumonia associada à ventilação mecânica.$^{16}$

Resultados semelhantes foram encontrados em duas UTI de um hospital público e de ensino em Teresina, em 2011, onde a distribuição topográfica das infecções hospitalares foi: $\quad 59,4 \%$ foram infecções respiratórias; $23,6 \%$ urinárias e 7,5\% da corrente sanguínea, dados que corroboram com o presente estudo. ${ }^{17}$

De acordo com os dados de vigilância do National Nosocomial Infection Surveillance (NNIS) do CDC, a pneumonia é a segunda infecção nosocomial em termos gerais e a infecção mais comum em UTI. Nos pacientes intubados, a incidência desta infecção é de 7 a 21 vezes maior do que aquele que não necessitam do ventilador. ${ }^{18}$

Foi verificado, em um estudo realizado em quatro UTI de FortalezaCE, a ocorrência de 202 casos de IRAS. Dentre as infecções notificadas, foi identificada a superioridade dos casos infecção respiratória, com $65,3 \%$ (sendo a grande maioria associada à VM); $17,8 \%$ foram IPCS e 16,9\% ITU. $^{19}$

Em 2007 foram internados 590 pacientes na UTI geral do Hospital Universitário Presidente Dutra de São Luís-MA, sendo que $81,3 \%$ submeteram-se à cateterização vesical de demora. Desses, 1,9\% apresentaram ITU, com uma média do tempo de internação de $39,3 \% .{ }^{20}$

Entre agosto de 2005 a janeiro de 2008, um estudo, conduzido em uma UTI de um hospital universitário brasileiro, apontou uma taxa de $\mathrm{IH}$ de $20 \%$ e aproximadamente $10 \%$ destes evoluíram a óbito, sendo a PAVM mais incidente. $^{12}$

Dados do NNIS apontam que as pneumonias somam aproximadamente $31 \%$ de todas as infecções em UTI, sendo para alguns autores o segundo sítio de infecção mais comum e a principal causa de morte dentre as infecções. ${ }^{21}$ Essa informação está em conformidade aos achados desta pesquisa.

Em um estudo realizado em uma UTI de um hospital universitário de Belo Horizonte, como desfecho dos 
pacientes, foi registrada uma taxa de mortalidade de $28,5 \%$, verificando-se que $47,5 \%$ dos pacientes, que evoluíram ao óbito, tiveram um diagnóstico de IRAS, e as infecções do trato respiratório foram as mais incidentes. ${ }^{22}$

A sepse é uma causa importante de hospitalização, e a principal causa de morte em UTI, trata-se de uma síndrome de resposta inflamatória, motivada por um agente agressor, associada à infecção sistêmica. Os sinais e sintomas apresentam de forma diversificada, sobretudo em pacientes graves, cujas doenças são complexas e com frequência já estão em uso de antimicrobianos. ${ }^{23}$

Em estudo acerca de resistência bacteriana realizado em Unidades de Terapia Intensiva, de 65 hospitais do Brasil foi verificado que a mortalidade global dos pacientes sépticos foi de 46,6\% (243 pacientes); em relação aos pacientes com choque séptico, a mortalidade encontrada foi de $65,3 \%$ e na sepse grave foi de $34,4 \% .{ }^{24}$

Dentro de um programa de controle de infecção, deve-se considerar a unidade de terapia intensiva como local prioritário de monitoramento e vigilância de IRAS, pois esta é uma manifestação frequente no paciente internado nessa unidade, representando grave risco de comprometimento.

Sendo assim, para que o controle da infecção relacionada à assistência à saúde seja realizado de forma sistemática, por todos que participam direta ou indiretamente do processo, e atenda às necessidades de cada serviço, políticas e normas deverão estabelecer conceitos e organizar estrutura física e humana e formas de trabalho, para com isso melhorar a qualidade da assistência prestada a população.

\section{Agradecimentos}

Esta pesquisa foi realizada com o apoio da Universidade Ceuma.

\section{Referências}

1. SANTOS, A.A.M; LOPES, F.F.P; CARDOSO, M.R.A; SERUFO, J.C. Diagnóstico do controle da infecção hospitalar no Brasil. Brasília (DF): Agência Nacional de Vigilância Sanitária, 2005.

2. HORAN, T.C; ANDRUS, M; DUDECK, M.A. CDC/NHSN Surveillance definition of health care-associated infection and criterio for specific types of infections in the acute care setting. Am J Infect Control. 2008; 36 (5): 311-31. doi: 10.1016/j.ajic.2008.03.002

3. KLEVENS, R.M; EDWARDS, J.R; RICHARDS, Jr. C.L et al. Estimating health care-associated infections and deaths in US hospitals, 2002. Public Health Rep. 2007; 122 (2): 160-6. Pmc1820440

4. LEISER, J.J; TOGNIM, M.C.B; BEDENDO, J. Infecções hospitalares em um Centro de Terapia Intensiva de um hospital de ensino no norte do Paraná. Cienc. Cuid. Saúde. 2007; 6 (2): 181-6. http://dx.doi.org/10.425/cienccuidsa ude.v6i2.4149

5. RUTALLA, A.W; WHITE, M.S; GERGEN, M.F; WEBER, D.J. Bacterial contamination of keyboards: efficacy and functional impact of disinfectants. Infect Control Hosp. Epidemiol. 2006, 27 (4):

372-7. http://dx.doi.org/10.1086/503340

6. WHO Guidelines on Hand Hygiene in Health Care. First Global Patient Safety Challenge Clean Care is Safer Care. World Health 
Organizations; 2009. ISBN: 9789241597906

GUIMARÃES, A.C; DONALISIO, M.R; SANTIAGO, T.H.R; FREIRE, J.B. Óbitos associados à infecção hospitalar ocorridos em um hospital geral de Sumaré-SP, Brasil. Rev. Brasileira Enf. 2011; 64 (5): 864-9. http://dx.doi.org/10.590/S0034716720113000500010

8. ALLEN, S. Prevention and Control of infection in the ICU. Curr Anaesth Crit. Care. 2005; 16 (4): 191-92. doi: 10.1016/J.idc.2009.04.012

9. RITCHIE, D.J; ALEXANDER, B.T; FINNEGAN, P.M. New Antimicrobial Agents for use in the Intensive Care Unit. Infect Dis Clin N. Am. 2009; 23 (3): 665-81. doi: 10.1016/j.idc.2009.04.010

10. FREEMAN, J; GOWAN, J.E. Risk factors nosocomial infections. Califórnia Baltimore, 2005. Disponível em $<$ www.revistamedicaanacosta.com. br>. Acesso em: 02 de agosto de 2015.

11. GUSMÃO, M.E.N. Infecção Hospitalar: mortalidade, sobrevida e fatores prognósticos no Hospital Universitário Professor Edgar Santos/UFBA. Tese de doutorado defendida em 29 de abril de 2008.

12. OLIVEIRA, A.C; KOVNER, C.T; SILVA, R.S. Infecção Hospitalar em unidade de tratamento intensivo de um hospital universitário brasileiro. Rev. Latino-Americana Enfermagem, 2010; 18 (2): [08 telas]. http://dx.doi.org/10.590/S0104116920010000200014

13. OLIVIER, C.A; BLAKE, R.K, STEED, L.L. Risk of vancomycin resistant Enterococcus bloodstream infection among colonized individuals. Infect Control Hosp. Epidemiol. 2008; 29 (5): 404-9. doi: 10.1016/j.bjid.2014.09.010

14. FIGUEIREDO, D.A. Fatores de risco associados à Infecção Hospitalar em uma Unidade de Terapia Intensiva. Dissertação de mestrado defendida em 08 de junho de 2012.

15. KAHVECI, F; ÕZAKIN,C; et al. Influences of therapy protocol and continous infections in ICU: disease consultation on antibiotic susceptibility. Washington: Intensive Care Med Publisher, 2009. doi: 10.1378/chest.118.1.146

16. SILVA, T.C; ALMEIDA, F.F; VERSIANI, C.C; et al. Infecções hospitalares relacionadas a procedimentos invasivos em um Centro de Terapia Intensiva adulta. Revista da Escola de Enfermagem da USP, vol.44, n.1, p.161-165; São Paulo, 2010. http://dx.doi.org/10.1590/S003471672007000400011

17. CARVALHO, M.M; MOURA, M.E.B; NUNES, M.R.C.M; et al. Infecções Hospitalares nas Unidades de Terapia Intensiva em um hospital público. Revista Interdisciplinar NOVAPAPI, Teresina, vol.4, n.4, p.42-48, 2011.

18. BERALDO, C.C; ANDRADE, D. Higiene bucal com clorexidina na prevenção de pneumonia associada à ventilação mecânica. Jornal Brasileiro de Pneumologia. 34 edição, 2008. http://dx.doi.org/10.1590/S180637132008000900012

19. BARROS, L.M; BENTO, J.N.C; CAETANO, J.A; et al. Prevalência de microrganismo e sensibilidade antimicrobiana de infecções hospitalares em unidade de terapia 
intensiva de hospital público no Brasil. Revista Ciência Farm. Básica Apl. 33 (3); 429-435, 2012.

20. OLIVEIRA, A.C.C; SILVA, A.C.O. Prevalência de infecção do trato urinário relacionada ao cateter vesical de demora em pacientes de UTI. Revista Pesquisa Saúde, 11 (1): 27-31, 2010. http://dx.doi.org/10.1590/S010443201999000100007

21. MESIANO, E.R.A.B; MERCHÃOHAMANN. Bloodstream Infections among patients using central venous catheters in intensive care units. Revista Latino Americana de Enfermagem 2007; 15 (3): 453-9. http://dx.doi.org/10.1590/S010411692007000300014

22. OLIVEIRA, A.C; PAULA, A.O; IQUIAPAZZA, R.A; LACERDA, A.C.S. Infecções relacionadas à assistência em saúde e gravidade clínica em uma unidade de terapia intensiva. Revista Gaúcha de Enfermagem, vol. 33, n. 3. Porto Alegre, 2012. http://dx.doi.org/10.1590/S198314472012000300012

23. BOECHAT, A.L; BOECHAT, N.O. Sepse: diagnóstico e tratamento. Revista Brasileira Clínica Médica. São Paulo, 2010; 8 (5): 420-7.

24. SALES, J.A.L; DAVID, C.M; HATUM, R; et al. Sepse Brasil: Estudo Epidemiológico da Sepse em Unidades de Terapia Intensiva brasileiras. Revista Brasileira de Terapia Intensiva, 2006; 18 (1): 917. http://dx.doi.org/10.1590/S0103507×200600100003 